\title{
Design sensitivity and shape optimization of geometrical nonlinear structure
}

\author{
Said Abid, Abdessalem Jarraya a , Fakhreddine Dammak and Mohamed Haddar \\ Mechanical, Modelisation and Manufacturing Unit (U2MP), National School of Engineers of Sfax (ENIS), \\ BP W 3038 Sfax, Tunisia
}

Received 1 April 2007, Accepted 17 October 2007

\begin{abstract}
This paper presents the structural shape optimization with linear elastic material, large displacement and small strains. The whole shape optimization process is carried out by coupling a closed geometric shape in $R^{2}$ with boundaries defined by B-splines curves, automatic mesh generation at each iteration, exact sensitivity analysis and mathematical programming method (SQP: Sequential Quadratic Programming). The design variables are the control point's coordinates which minimize the Von-Mises criteria, with a constraint that the total material volume of the structure remains constant. In this work the sensitivity analysis is performed using two methods: numerically by an efficient finite difference scheme and by the new method called exact Jacobian method. The feasibility of the proposed method is carried out for two numerical examples.
\end{abstract}

Key words: Geometrical nonlinearity / finite-elements / shape optimization / parameterization / sensitivity analysis / SQP method

\begin{abstract}
Résumé - Optimisation de forme des structures en présence de non-linéarité géometrique avec un calcul exact du jacobien. Dans ce travail, nous nous sommes intéressés à l'optimisation de forme des structures en présence de non-linéarités géométriques (grands déplacements). Les variables d'optimisation sont les coordonnées des points de contrôle avec une paramétrisation par les courbes de B-splines. Le critère d'optimisation (fonction objectif à minimiser) est défini à partir du critère de Von Mises avec conservation du volume de la structure le long du processus d'optimisation. La résolution du problème mécanique est faite par la méthode des éléments-finis. L'algorithme d'optimisation utilisé est (S.Q.P. : séquential quadratic programming). Cet article présente une description détaillée du calcul de la sensibilité par la méthode exacte et la méthode différence finie pour un matériau à comportement élastique isotrope. Deux exemples numériques seront réalisés.
\end{abstract}

Mots clés : Optimisation de forme / non-linéarités géométriques / éléments-finis / sensibilité exacte / paramétrisation méthode S.Q.P.

\section{Introduction}

Structural design optimization is a critical and a challenging activity that has received considerable attention in the last two decades. Designers are able to produce better designs while saving time and money through optimization. Traditionally, various mathematical methods such as linear or nonlinear programming have been developed to solve engineering optimization problems.

Many academic and commercial softwares incorporate shape optimization modules of linear structures in

\footnotetext{
a Corresponding author:

jarraya_abdessalem@yahoo.fr
}

the various fields of mechanics (static, dynamic, thermal...). These modules are rather reliable, but always require precautions at the time of the problem modeling to be treated (parameterization, choice of the optimization variables...).

Many works have been made in recent years; as those proposed by Canales et al. [1] where they have developed an integrated system for shape optimal design using a parametric geometric model and adaptive mesh refinement.

Younsi [2] have presented the shape optimization of three-dimensional structures; the sensitivity has been computed by using finite difference method. Naceur [3] 
have treated the shape optimization of thin structures (beams shells) in the presence of geometrical and nonlinear material. The sensitivity analysis has been computed by the exact Jacobian method only for a beam and axisymmetric structure.

Dems \& Haftka [4] have presented two approaches for sensitivity analysis considering the shape variation of structures. Haftka \& Adelman [5] have treated recent developments in structural sensitivity analysis structural optimization. Srikanth \& Zabaras [6] have presented the shape optimization and perform design in metal forming processes that a continuum sensitivity analysis is presented for the computation of the shape sensitivity of finite structure deformations involving contact with friction and using a direct differentiation method. David \& Phelan [7] have treated an adjoint variable method for sensitivity analysis of nonlinear elastic systems where the mutual energy expression used in the adjoint sensitivity derivation from a nonlinear extension of the $\mathrm{Hu}$-Washizu energy functional and yields a linear governing equation for the adjoint systems. Yatheendhar \& Belegundu [8] have developed analytical shape sensitivity by implicit differentiation for general velocity fields that the shape sensitivity is derived with the exact method for linear structure systems. Kim \& Choi [9] have proposed a shape design sensitivity analysis (DSA) and optimization of structural transient dynamics for the finite deformation elastoplastic materials under impact with a rigid surface. A shape variation of the structure is considered using the material derivative approach in continuum mechanics. The design sensitivity equation is solved at each converged time step with the same tangent stiffness matrix as response analysis without iteration. The cost of sensitivity computation is more efficient than the cost of response analysis for the implicit time integration method. Song \& Baldwin [10] have developed a novel node-based shape optimization algorithm. The method proposed put the maximum weights on the selected boundary nodes, referred to as the design points, so that the time consuming sensitivity analysis is based on the perturbation of only these nodes. Rohan \& Whiteman [11] have treated the sensitivity analysis and optimization of elasto-plastic bodies when isotropic strain hardening takes place. The elasto-plastic behaviour of the material is governed by a nonlinear complementarities problem. The sensitivity analysis based on the adjoint-variable technique is derived for a history-dependent problem.

Kim et al. [12] have developed design sensitivity analysis of a sequential structural-acoustic problem. The design sensitivity analysis, is computed with a direct differentiation method and an adjoint variable method. In this method, an adjoint load is obtained from the acoustic boundary element re-analysis, while the adjoint solution is calculated from the structural dynamic re-analysis. The evaluation of pressure sensitivity involves only a numerical integration process for the structural part. The proposed sensitivity results are compared to those given by finite difference calculation with excellent agreement. Oral [13] have presented a hybrid-stress Mindlin plate finite element and its sensitivity derivatives. Analytical and semi-analytical methods for the thickness and shape design variables are used to compute sensitivity analysis. Tanaka \& Naguchi [14] have proposed a new method based on the idea developed by Azegami \& Wu [15] to handle any kinds of nonlinearity. This method treated the structural shape optimization of hyperelastic material by discrete force.

Uysala et al. [16] have treated the shape optimization for three-dimensional shell structures. The shape optimization program is implemented by a job control language and a reliable finite-element package program, ANSYS, is used for structural analysis. The objective is to minimize the weight of the shell structure under constraints that are the maximum value of the Von Mises stress in each element and the move limit for each design variable. The design sensitivities are calculated using the finite difference method. The search for the final shape of a structure is performed using the linear programming technique.

To obtain a better structural design by change the shape of the structure, (DSA) is a critical step. Haug et al. [17] have proved the existence of sensitivity for linear elastic structural systems and derived shape sensitivity formulation based on a continuum approach. No mathematical proof is available for the existence or uniqueness of the shape sensitivity for a nonlinear structure. Under the regularity assumption, Santos \& Choi [18] have derived shape sensitivity of nonlinear elastic materials. Grindeanu et al. [19] have developed nonlinear shape design sensitivity formulation using the free mesh method. The design sensitivity equation is obtained by taking the material derivative of the variational equation with respect to the shape design parameters at the final converged configuration.

In the present work, the shape optimization with linear elastic material, large displacement, and small strains is treated. The nonlinear structural problem is performed by means of the finite-element method. Firstly, we present the design variables which are the coordinates of the B-spline curves. Secondly, we determine the objective function that presents the Von Mises constraints with conservation of the volume. Finally, we develop an exact sensitivity analysis. In order to assess the performance of this development, we present two numerical examples including large displacements and small strains.

\section{Design variables and shape parameterization}

In shape optimization, coordinates of certain structural nodes can be selected as design variables. In order to allow a smooth and efficient solution, the number of design variables should be kept as small as possible but still allowing enough freedom for general shape. We can present the shape evolution by interpolation of the B-spline type. This method consists in dividing a curve of data into a certain number of segments, each one being 
$\mathrm{d}_{5}$

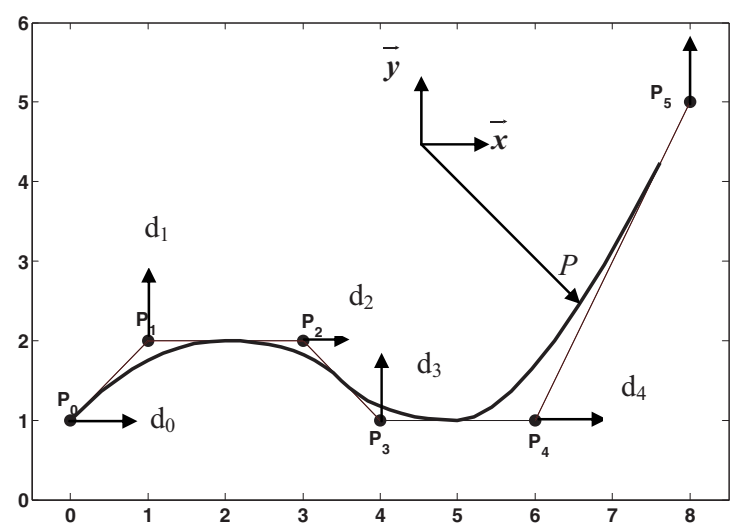

$\mathrm{d}_{0}(1,0)$

$\mathrm{d}_{1}(0,1)$

$\mathrm{d}_{2}(1,0)$

$\mathrm{d}_{3}(0,1)$

$\mathrm{d}_{4}(1,0)$

$\mathrm{d}_{5}(0,1)$

Fig. 1. B-spline curve with 6 controls points.

defined by polynomials of B-spline, and to connect these segments to form a made up curve (Fig. 1). The connection of the segments is made by observing conditions of continuity to the control nodes. Since these polynomials have a displacement local support, point of control modifies locally the shape of the curve, i.e. in a vicinity of this point. For this reason, B-spline is frequently used in optimization.

Letting $\boldsymbol{P}(t)$ to be the position vectors along the curve as a function of the parameter $t$, a B-spline curve is given by [20]

$$
\boldsymbol{P}(t)=\sum_{i=1}^{n+1} \boldsymbol{P}_{i} N_{i, k}(t) \quad t_{\min } \leqslant t \leqslant t_{\max }, \quad 2 \leqslant k \leqslant n+1
$$

where $\boldsymbol{P}_{i}$ is the position vector of the $n+1$ defining polygon vertices and $N_{i, k}$ are the normalized B-spline basis functions.

For the $i$ th normalized B-spline basis function of order $k$ (degree $k-1)$, the basis functions $N_{i, k}(t)$ are defined by the recursion formulas.

Specifically,

$$
N_{i, 1}(t)= \begin{cases}1 & \text { if } x_{i} \leqslant t \leqslant x_{i+1} \\ 0 & \text { otherwise }\end{cases}
$$

and

$$
N_{i, k}(t)=\frac{\left(t-x_{i}\right) N_{i, k-1}(t)}{x_{i+k-1}-x_{i}}+\frac{\left(x_{i+k}-t\right) N_{i+1, k-1}(t)}{x_{i+k}-x_{i+1}}
$$

The values of $x_{i}$ are elements of a knot vector satisfying the relation $x_{i} \leqslant x_{i+1}$, the parameter $t$ varies from $t_{\text {min }}$ to $t_{\max }$ along the curve $\boldsymbol{P}(t)$.

\section{Objective function and constraints}

Generally, an optimization problem with limitations (constraints) is written in the following form:

$$
\text { Minimize } f(v, \boldsymbol{U}), \quad \boldsymbol{v} \in \boldsymbol{R}^{n}
$$

Subject to a set of $m$ limitations.

$$
\begin{array}{ll}
g_{i}(\boldsymbol{v})=0 & i=1, \ldots, m^{\prime} \\
g_{i}(\boldsymbol{v}) \leqslant 0 & i=m^{\prime}, \ldots, m
\end{array}
$$

where $\boldsymbol{v}$ is the design variables vector and $\boldsymbol{U}$ is the displacement vector.

The cost function $f(\boldsymbol{v}, \boldsymbol{U})$ and limitations $g_{i}(\boldsymbol{v})$ are considered differentiable. The optimal solution of the problem can be done, generally, only by a numerical way.

Our principal goal through the optimization process (from the mechanical design point of view) is to seek the optimal shape of the average line. This makes it possible to reduce the maximum of Von Mises constraints in the structure, while satisfying certain geometrical limitations. By taking into account all these concerns, we developed a criterion of optimization which is a continuous and differentiable function. The objective function selected in this work is based on the Von Mises criteria

$$
f(\boldsymbol{v}, \boldsymbol{U})=\frac{1}{2} \int \boldsymbol{S}_{\mathrm{eq}}^{2} \mathrm{~d} V
$$

where $\boldsymbol{S}_{\text {eq }}$ is the Von Mises criteria written as a function of the Piola-Kirchoff stress tensor components.

Under limitations which consist to conserve the volume of the structure before and after optimization:

$$
g=\int_{V} \mathrm{~d} V-V_{0}=0
$$

\section{Sensitivity analysis}

The sensitivity analysis supplies gradient information on objective and constraint functions with respect to optimization variables. In general any function $f$ (objective or constraint) depends on design variables $v$ and state variables $\boldsymbol{U}$ (displacements). Thus, the total derivative of the function $f$ is given as

$$
\frac{\mathrm{d} f}{\mathrm{~d} v}=\frac{\partial f}{\partial v}+\frac{\partial f}{\partial \boldsymbol{U}} \cdot \frac{\partial \boldsymbol{U}}{\partial v}
$$

where the determination of the response sensitivity, $\frac{\partial U}{\partial v}$, is part of job. It can be carried out by several different techniques (numerical or analytical). It is also known that the partial derivatives of the functions with respect to the global displacement $\left(\frac{\partial f}{\partial \boldsymbol{U}}\right)$ are then required. This derivative can be easily evaluated when the functions are explicitly written in terms of displacements.

In this work the discrete design sensitivity analysis is applied in which the gradients are obtained after discretization. Here two approaches are used. In the numerical one, each component of the gradients is evaluated by finite difference. Opposite to this method the analytic method requires analytical derivatives of stiffness matrix, load vector and stress with respect to the design parameters.

In the following of this section we concentrate on the analytic method concerning the constraint function $g$ which represents the total volume of the structure and the objective function expressed on terms of stress components. 


\subsection{Volume derivative}

After the discretization, the total volume of the structure is given by

$$
V=\int_{V} \mathrm{~d} V=\sum_{e=1}^{n_{\text {elt }}} V_{e}=\sum_{e=1}^{n_{\text {elt }}} \int_{V_{e}} \mathrm{~d} V_{e}
$$

where $n_{\text {elt }}$ is the total number of the finite-elements, $\mathrm{d} V_{e}=\operatorname{det} \boldsymbol{J} . \mathrm{d} V_{\xi}$ is the finite-element volume, $\boldsymbol{J}$ is the Jacobian matrix and $\mathrm{d} V_{\xi}=\mathrm{d} \xi \mathrm{d} \eta$ in two dimensional is the reference volume element.

The element volume becomes

$$
V_{e}=\int_{V_{\xi}} \operatorname{det} \boldsymbol{J} \mathrm{d} V_{\xi}
$$

It is clear that the element volume is independent of the displacement vector, that means the total derivative of Equation (10) with respect to any design variable $v_{i}$ is readily obtained as

$$
\frac{\mathrm{d} V_{e}}{\mathrm{~d} V_{i}}=\frac{\partial V_{e}}{\partial v_{i}}=\int_{V_{\xi}} \frac{\partial \operatorname{det} \boldsymbol{J}}{\partial v_{i}} \mathrm{~d} V_{\xi}
$$

where

$$
\frac{\partial \operatorname{det} \boldsymbol{J}}{\partial v_{i}}=J_{11} \frac{\partial J_{22}}{\partial v_{i}}+J_{22} \frac{\partial J_{11}}{\partial v_{i}}-J_{12} \frac{\partial J_{21}}{\partial v_{i}}-J_{21} \frac{\partial J_{12}}{\partial v_{i}}
$$

\subsection{Stress derivative}

The discrete form of the objective function is given by

$$
f=\sum_{e=1}^{n_{\text {elt }}} f_{\mathrm{e}}=\sum_{e=1}^{n_{\text {elt }}} \frac{1}{2} \int_{V_{e}} S_{\text {eq }}^{2} \mathrm{~d} V=\sum_{e=1}^{n_{\text {elt }}} \frac{1}{2} \int_{V_{\xi}} S_{\text {eq }}^{2} \operatorname{det} \boldsymbol{J} \mathrm{d} V_{\xi}
$$

By using Equation (8), the total derivative of the elementary function $f_{\mathrm{e}}$ with respect to any design variable $v_{i}$ is given as

$$
\frac{\mathrm{d} f_{\mathrm{e}}}{\mathrm{d} v_{i}}=\frac{\partial f_{\mathrm{e}}}{\partial v_{i}}+\frac{\partial f_{\mathrm{e}}}{\partial \boldsymbol{U}} \cdot \frac{\partial \boldsymbol{U}}{\partial v_{i}}
$$

Equation (14) contains three derivatives that must be evaluated. First consider $\frac{\partial f_{\mathrm{e}}}{\partial v_{i}}$

$$
\frac{\partial f_{\mathrm{e}}}{\partial v_{i}}=\int_{V_{\xi}}\left[S_{\mathrm{eq}} \frac{\partial S_{\mathrm{eq}}}{\partial v_{i}} \operatorname{det} \boldsymbol{J}+\frac{1}{2} S_{\text {eq }}^{2} \frac{\partial \operatorname{det} \boldsymbol{J}}{\partial v_{i}}\right] \mathrm{d} V_{\xi}
$$

The unknown term of Equation (15) is $\frac{\partial S_{\mathrm{eq}}}{\partial v_{i}}$ which represents the sensitivity of the Von Mises criteria to the design variable. This term can be easily computed by using the second Piola-Kirchoff stress tensor

$$
\boldsymbol{S}=£ .: \boldsymbol{E}
$$

where $\boldsymbol{E}$ and $£$ are respectively the Green-Lagrange strain tensor and the elastic tensor. The Green-Lagrange strain tensor is given by

$$
E=(C-I) / 2
$$

where $\boldsymbol{C}$ is the Cauchy-Green strain tensor which may be written by using the strain gradient tensor $\boldsymbol{F}$

$$
\boldsymbol{C}=\boldsymbol{F}^{T} \boldsymbol{F}
$$

The derivative of the second Piola-Kirchoff stress tensor with respect to the design variable $v_{i}$ is found to be

$$
\frac{\partial \boldsymbol{S}}{\partial v_{i}}=\frac{1}{2} £: \frac{\partial \boldsymbol{C}}{\partial v_{i}}
$$

where

$$
\frac{\partial \boldsymbol{C}}{\partial v_{i}}=\frac{\partial \boldsymbol{F}^{T}}{\partial v_{i}} \boldsymbol{F}+\frac{\partial \boldsymbol{F}}{\partial v_{i}} \boldsymbol{F}^{T}
$$

By using Equation (19), the term $\frac{\partial S_{\mathrm{eq}}}{\partial v_{i}}$ can be computed.

The partial derivatives of the objective function with respect to the global displacement $\frac{\partial f_{\mathrm{e}}}{\partial \boldsymbol{U}}$ (second unknown derivative term of Eq. (14)) is given by

$$
\frac{\partial f}{\partial \boldsymbol{U}}=\int_{V_{\xi}} S_{\mathrm{eq}} \frac{\partial S_{\mathrm{eq}}}{\partial \boldsymbol{U}} \operatorname{det} \boldsymbol{J} \mathrm{d} V_{\xi}
$$

Using the same procedure as before (19) we obtain

$$
\frac{\partial \boldsymbol{S}}{\partial \boldsymbol{U}}=\frac{1}{2} £: \frac{\partial \boldsymbol{C}}{\partial \boldsymbol{U}}
$$

where

$$
\frac{\partial \boldsymbol{C}}{\partial \boldsymbol{U}}=\frac{\partial \boldsymbol{F}^{T}}{\partial \boldsymbol{U}} \boldsymbol{F}+\boldsymbol{F}^{T} \frac{\partial \boldsymbol{F}}{\partial \boldsymbol{U}}
$$

Equation (23) is used to compute $\frac{\partial S_{\mathrm{eq}}}{\partial \boldsymbol{U}}$.

The third derivative term of Equation (14) which represents the response sensitivity $\frac{\partial \boldsymbol{U}}{\partial v_{i}}$ can be computed by using the equilibrium equation of the interior and external forces

$$
\boldsymbol{F}_{\text {int }}-\boldsymbol{F}_{\text {ext }}=\mathbf{0}
$$

The derivative of Equation (24) with respect to the design variable $v_{i}$ is given by

$$
\frac{\mathrm{d} \boldsymbol{F}_{\text {int }}}{\mathrm{d} v_{i}}-\frac{\mathrm{d} \boldsymbol{F}_{\text {ext }}}{\mathrm{d} v_{i}}=\mathbf{0}
$$

The derivative, $\frac{\mathrm{d} \boldsymbol{F}_{\text {ext }}}{\mathrm{d} v_{i}}$, represents the sensitivity of the applied forces to the design variables. In many cases the applied forces are independent of the design variables and this term is equal to zero. Thus Equation (25) becomes

$$
\frac{\mathrm{d} \boldsymbol{F}_{\text {int }}}{\mathrm{d} v_{i}}=\frac{\partial \boldsymbol{F}_{\text {int }}}{\partial v_{i}}+\frac{\partial \boldsymbol{F}_{\text {int }}}{\partial \boldsymbol{U}} \frac{\partial \boldsymbol{U}}{\partial v_{i}}=\mathbf{0}
$$

where $\frac{\partial \boldsymbol{F}_{\text {int }}}{\partial \boldsymbol{U}}$ can be identify as the global tangent stiffness matrix

$$
\frac{\partial \boldsymbol{F}_{\text {int }}}{\partial \boldsymbol{U}}=\boldsymbol{K}_{T}
$$

Substituting Equation (27) into (26)

$$
\boldsymbol{K}_{T} \frac{\partial U}{\partial v_{i}}=-\frac{\partial \boldsymbol{F}_{\text {int }}}{\partial v_{i}}
$$




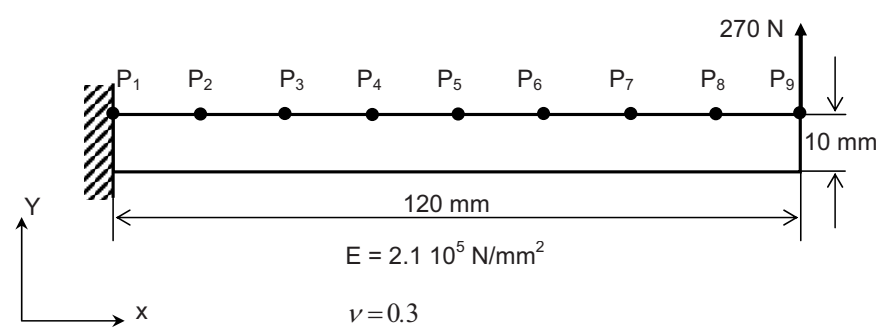

Fig. 2. Cantilever beam. Initial geometry, control points and mechanical property.

The term on the right side of Equation (28) represents the derivative of the internal vector residue with respect to the design variable.

After discretization, the internal vector force is given by

$\boldsymbol{F}_{\text {int }}=\sum_{e=1}^{n_{\text {elt }}} \boldsymbol{F}_{\text {int }}^{e}=\sum_{e=1}^{n_{\text {elt }}} \int_{V_{e}} \boldsymbol{B}^{T} S \mathrm{~d} V=\sum_{e=1}^{n_{\text {elt }}} \int_{V_{\xi}} \boldsymbol{B}^{T} \boldsymbol{S} \operatorname{det} \boldsymbol{J} \mathrm{d} V_{\xi}$

where $\boldsymbol{B}$ is the element strain displacement matrix.

The derivative of the elementary internal vector force with respect to the design variable $v_{i}$ is evaluated by

$$
\begin{array}{r}
\frac{\partial \boldsymbol{F}_{\text {int }}^{e}}{\partial v_{i}}=\int_{V_{\xi}}\left[\boldsymbol{B}^{T}\left(\frac{\partial \boldsymbol{S}}{\partial v_{i}} \operatorname{det} \boldsymbol{J}+\boldsymbol{S} \frac{\partial \operatorname{det} \boldsymbol{J}}{\partial v_{i}}\right)\right. \\
\left.+\left(\frac{\partial \boldsymbol{B}}{\partial v_{i}}\right)^{T} \boldsymbol{S} \operatorname{det} \boldsymbol{J}\right] \mathrm{d} V_{\xi}
\end{array}
$$

By the resolution of Equation (28), the term $\frac{\partial \boldsymbol{U}}{\partial v_{i}}$ can be computed.

\section{Implementation and numerical results}

To study the accuracy and the efficiency of the developed method in the previous sections, the algorithm was implemented and several examples are analyzed. The sensitivity analysis (numerical and analytical methods) was incorporated into a finite-element code. In this code the structural response can be considered linear or nonlinear. An automatic mesh generation was used at each iteration of the optimization process. The first example deals with a structure clumped at one side, the second is a portal frame. In the two tests suggested, the discretization was realized by the triangular finite-element with three nodes and two degrees of freedom per node.

\subsection{Example 1: Cantilever beam}

As first example, a simple cantilever beam is considered. The geometry, loading and material properties of the beam modeled are shown in Figure 2. The $270 \mathrm{~N}$ load is applied to the right end of the beam. The structure is discretized by 390 three-node finite-elements. To obtain

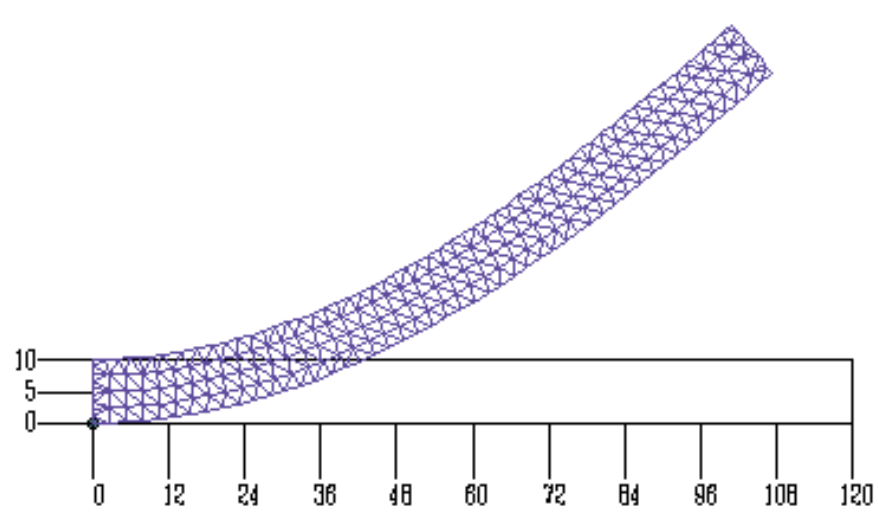

Fig. 3. Deformed structure before optimization.

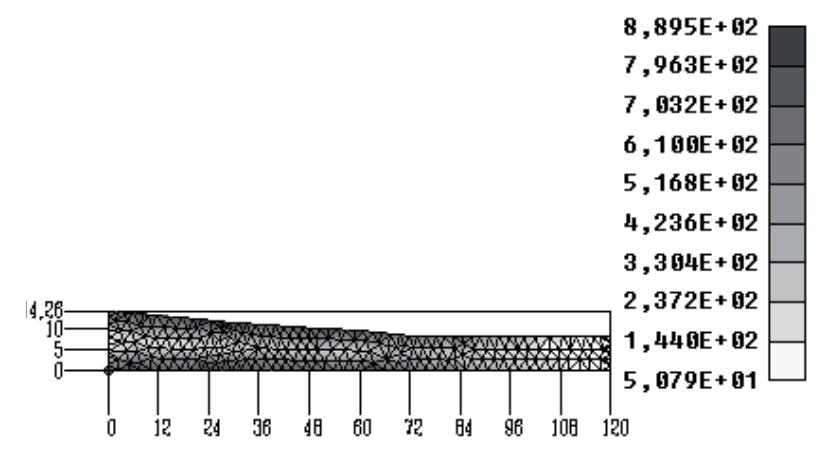

(a) Linear structural response

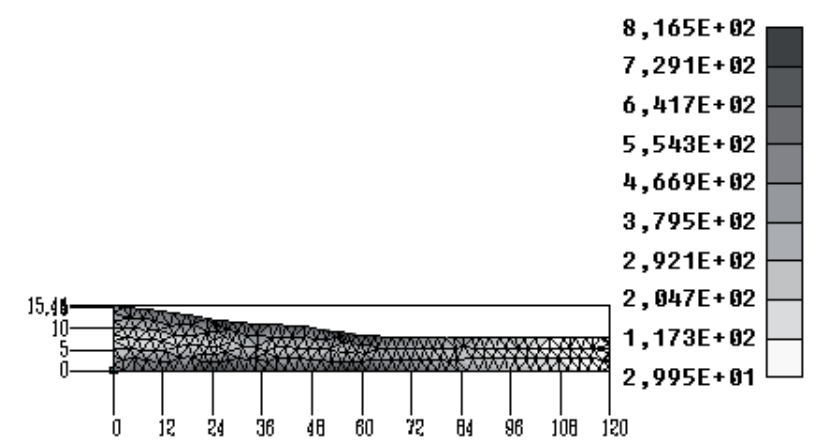

(b) Nonlinear structural response

Fig. 4. Optimal shapes.

the optimized shape, nine control points $\left(\boldsymbol{P}_{i} i=1, \ldots, 9\right)$ are used as design variables. The deformed shape of the structure before optimization shows an important nonlinearity of the problem (Fig. 3).

The different optimal shapes obtained when considering a linear and nonlinear structural response are displayed in Figure 4. In Figure 5 the iteration history of the objective function $\left(\frac{f}{f_{0}} \times 100\right.$ where $f_{0}$ is the initial value of the objective function) is presented. Optimization assuming a linear structural response results in a $15 \%$ reduction of the objective function; however optimization with a geometrical nonlinear response reduces the objective function by $20 \%$ from the nominal design. These results indicate that the problem is physically nonlinear. In Figure 6 the load as a function of displacement before 


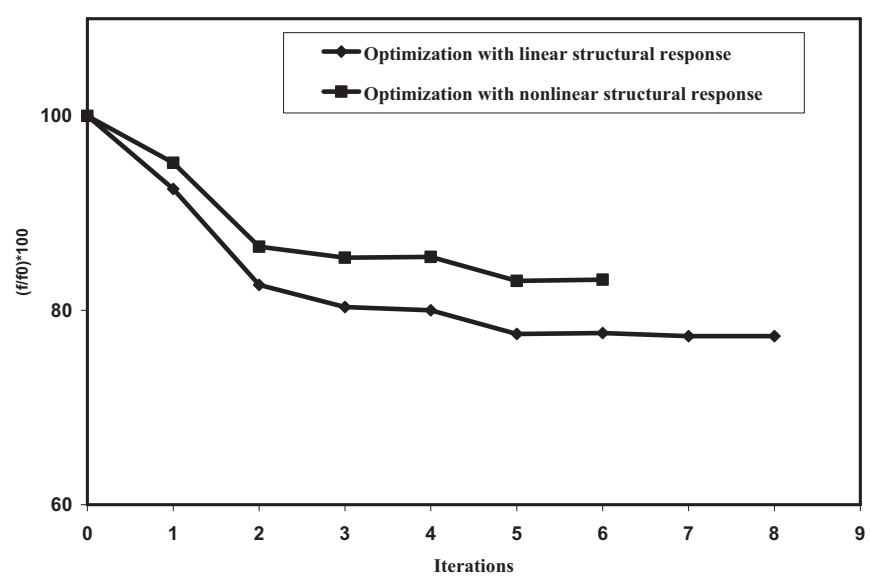

Fig. 5. Iteration history.

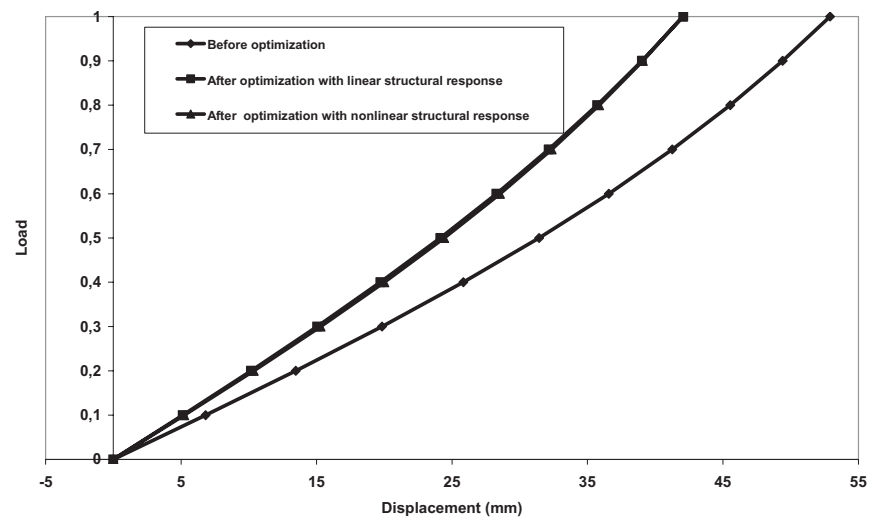

Fig. 6. Load as a function of displacement at $P_{9}$.

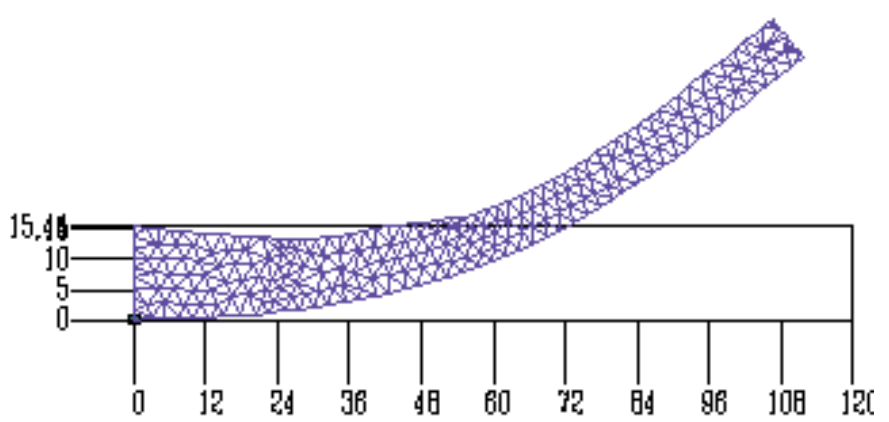

Fig. 7. Deformed structure after optimization.

and after optimization is presented. Figure 7 contains the deformed shape for nonlinear structural response after optimization.

\subsection{Example 2: Portal frame}

A simple portal frame is considered as a second example. The geometry of the structure, loading and material properties are shown in Figure 8. The external loading of $20 \mathrm{~N}$ is applied to the top side. The design variables are represented by six control points. Because of symmetry, only one half of the structure has been modeled using 292 three nodes finite elements. The initial mesh and Von Mises stress before optimization are presented
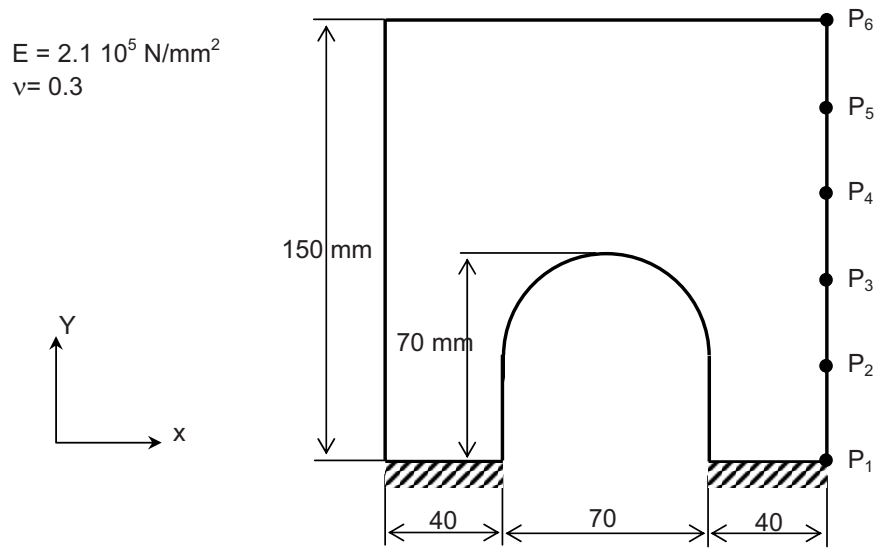

Fig. 8. Portal frame: initial geometry and control points.

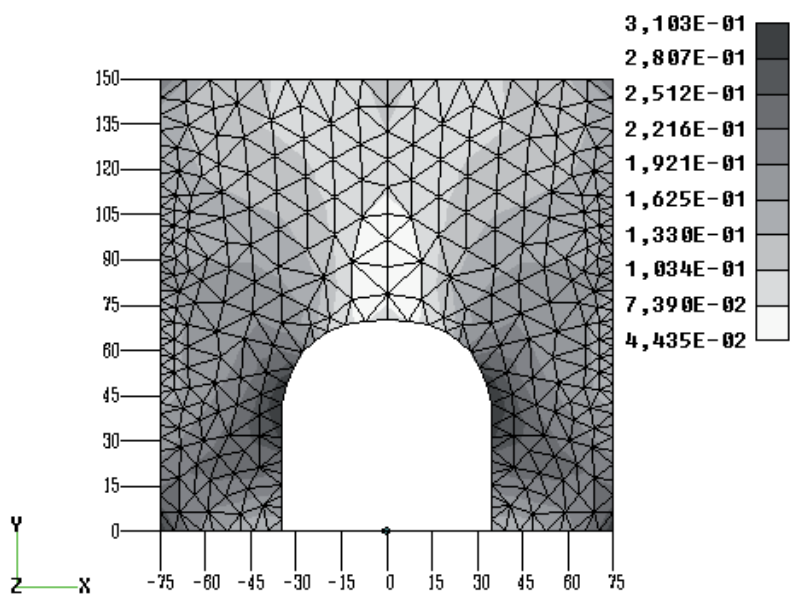

Fig. 9. Initial mesh and Von Mises stress before optimization.

in Figure 9. In this example the nonlinear structural response is only considered. Figure 10 presents the optimum design where sensitivity derivatives are obtained by the exact Jacobian method. The different iteration history of the objective function obtained when sensitivity analysis is computed by finite difference method and exact Jacobian method are displayed in Figure 11. Eight iterations are needed to obtain the optimal shape when finite difference method is used, however only five iterations are needed when the exact method is used. These results indicate that computing time of the exact Jacobian has an important reduction.

\section{Conclusions}

In this work we have presented the shape optimization of an elastic structure undergoing large displacements. Several results have been obtained for two examples, showing that our global approach is very satisfactory. This approach is based on:

- a definition of the objective function in terms of the Von Mises criteria,

- a simple and efficient analytic sensitivity analysis method called exact Jacobian method, 


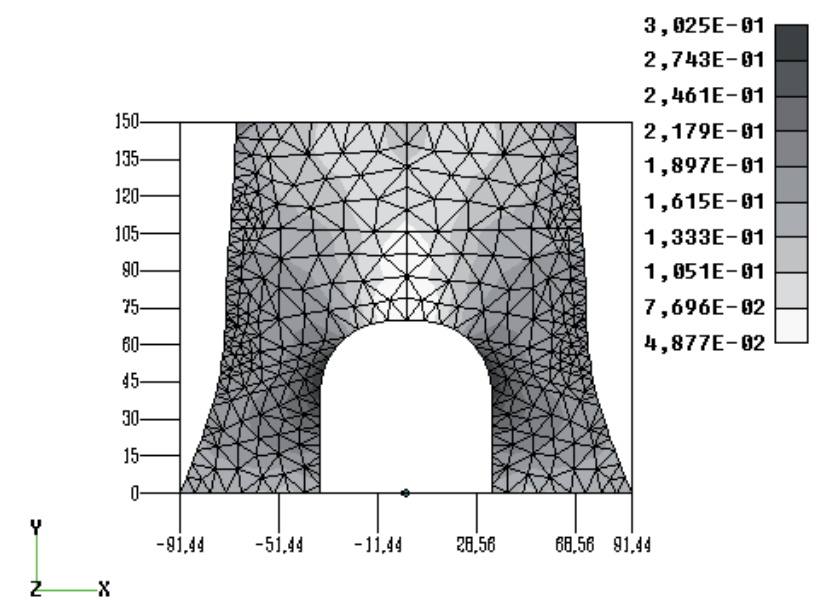

Fig. 10. Shape and Von Mises stress after optimization.

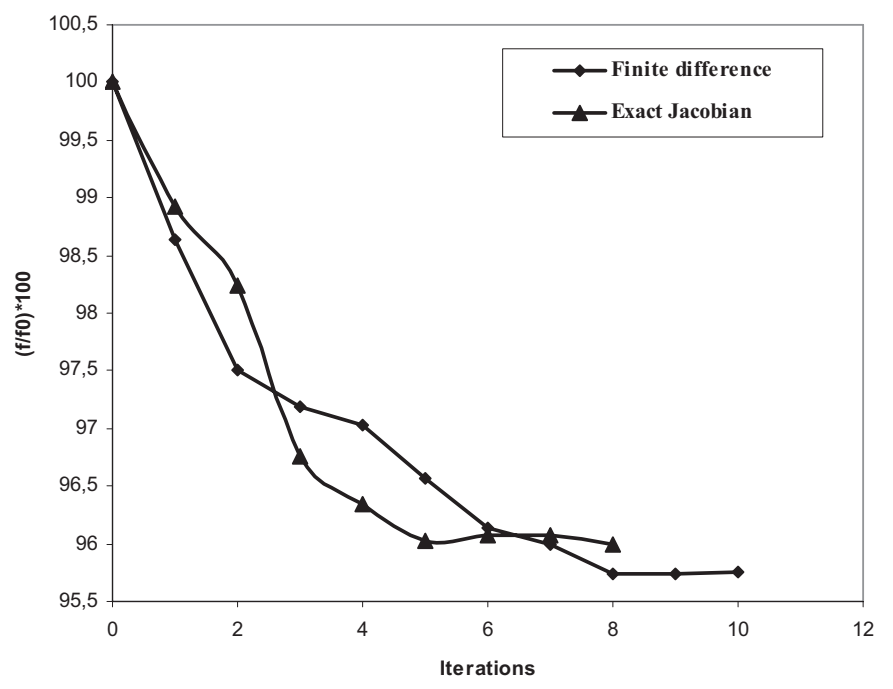

Fig. 11. Iteration history.

- a Sequential Quadratic Programming algorithm to solve the optimization problem,

- a shape parameterization by using B-splines functions involving a small number of control variables,

- an automatic mesh generation at each iteration of optimization.

In term of this work, we believe that developed tools can be used for analysis and design of new two dimensional structures with geometrical nonlinearities. The effects of material nonlinearities as well as time dependent influences have not be considered but are challenging task for further studies.

\section{References}

[1] J. Canales, A. Hernandez, M.F. Izaguirre, J.A. Tarrago, An integrated system for shape optimal design using a parametric geometric model and adaptive mesh refinement STRUCOME, 1992, 621-632
[2] R. Younsi, Optimisation de forme de structures tridimensionnelles, Thèse de Doctorat de l'Université de Technologie de Compiègne, 1993

[3] H. Naceur, Contribution à l'optimisation de forme de structures minces en présence de non linéarités géométriques et matérielles, Thèse de Doctorat de l'Université de Technologie de Compiègne, 1998

[4] K. Dems, R.T. Haftka, Two approaches to sensitivity analysis, for shape variation of structures, Mech. Struct. Mach 16 (1988) 501-522

[5] R.T. Haftka, H.L.M. Adelman, Recent developments in structural sensitivity analysis, Structural Optimization 1 (1989) 137-151

[6] A. Srikanth, N. Zabaras, Shape optimization and preform design in metal forming processes, Comput. Methods Appl. Mech. Engrg. 190 (2000) 1859-1901

[7] G. David, Phelan, An adjoint variable method for sensitivity analysis of nonlinear elastic systems, Int. J. numerical methods in engineering (1991) 1649-1667

[8] M.Yatheendhar, A.D. Belegundu, Analytical shape sensitivity by implicit differentiation for general velocity fields, Comput. Structures 46 (1993) 617-623

[9] N.H. Kim, K.K. Choi, Design sensitivity analysis and optimization of nonlinear transient dynamics, Mech. Structures and Machines 29 (2001) 351-371

[10] X. Song, J.D. Baldwin, A novel node-based structural shape optimization algorithm Computers and structures 70 (1999) 569-581

[11] E. Rohan, J.R. Whiteman, Shape optimization of elastoplastic structures and continua Comput. Methods Appl. Mech. Engrg. 187 (2000) 261-288

[12] N.H. Kim, J. Dong, K.K. Choi, N. Vlahopoulos, Z.D. Ma, M.P. Castanier, C. Pierre, Design sensitivity analysis for a sequential structural-acoustic problem, J. Sound Vib. 263 (2003) 569-591

[13] S. Oral, A Mindlin plate finite element with semianalytical shape design sensitivities, Computers and Structures 78 (2000) 467-472

[14] M. Tanaka, H. Noguchi, Structural shape optimization of hyperelastic material by discrete force method, Theoret. App. Mech. Japan 53 (2004) 83-91

[15] H. Azegami, Z.C. Wu, Domain optimization analysis in linear elastic problems: Approach using traction method, JSME Int. J., Se. A: Mech. Mat. Eng. 39 (1996) 272-278

[16] H. Uysala, R. Gula, U. Uzmanb, Optimum shape design of shell structures, Eng. Structures 29 (2007) 80-87

[17] E.J. Haug, K.K. Choi, V. Komkov, Design sensitivity analysis of structural systems, New York: Academic Press, 1986

[18] J.L.T. Santos, K.K. Choi, Shape design sensitivity analysis of nonlinear structural systems, Struct. Optim. 4 (1992) 23-35

[19] I. Grindeanu, K.H. Chang, K.K. Choi, J.S. Chen, Design sensitivity analysis of hyper-elastic structures using a meshless method, AIAA J. 36 (1987) 618-628

[20] D.F. Rogers, J.A. Adams, Mathematical elements for computer graphics, McGraw-Hill, 1990 